\title{
Increased efficacy of CDDP in a xenograft model of hepatoblastoma using the apoptosis sensitizer ABT-737
}

\author{
JUSTUS LIEBER, ALEXANDER DEWERTH, JULIA WENZ, BETTINA KIRCHNER, CARMEN EICHER, \\ STEVEN W. WARMANN, JÖRG FUCHS and SORIN ARMEANU-EBINGER
}

\author{
Department of Paediatric Surgery and Paediatric Urology, \\ University Children's Hospital, 72076 Tübingen, Germany
}

Received September 10, 2012; Accepted October 19, 2012

DOI: $10.3892 /$ or.2012.2150

\begin{abstract}
The response of standard-risk hepatoblastoma (HB) to neoadjuvant cisplatin (CDDP) chemotherapy is excellent; however, in high-risk $\mathrm{HB}$, drug resistance remains a major challenge. Alternative therapeutic strategies may consider combining cytotoxic drugs with apoptosis sensitizers as this has shown additive effects in various types of malignancies. Analysis of published expression databases have revealed an anti-apoptosis state in HB samples. Herein, we evaluated the synergistic effects of ABT-737 as a modulator of apoptosis in combination with CDDP in HB. To this end, clonogenic assays were performed with HepT1 and HUH6 HB cells to evaluate the synergistic effects of CDDP and ABT-737. Combination treatment with CDDP and ABT-737 reduced the clonogenicity of $\mathrm{HB}$ cells more than 5-fold compared to treatment with CDDP alone. Furthermore, the HUH6 mixed-type HB cells showed higher sensitivity to CDDP and combination treatment compared to the HepT1 embryonal-type cells. Subcutaneous HUH6 tumors in NOD/LtSz-scid IL2R $\gamma$ null mice were treated with CDDP (1.25 and $3 \mathrm{mg} / \mathrm{kg}$ body weight, $\mathrm{n}=6)$, ABT-737 (100 mg/kg, $n=5)$ and the combination of both agents $(n=5)$. Combined treatment led to a significantly reduced tumor growth compared to CDDP treatment alone $(\mathrm{p}<0.02)$. When using higher doses of CDDP (3 mg/kg) alone or in combination with ABT-737, dose-dependent toxicity was observed in this mouse strain. In conclusion, our results demonstrated the enhancement of chemotherapy efficacy by using modulators of apoptosis together with cytotoxic agents. Additive effects of ABT-737
\end{abstract}

Correspondence to: Dr Justus Lieber, Department of Paediatric Surgery and Paediatric Urology, University Children's Hospital, Hoppe-Seyler-Strasse 1, 72076 Tübingen, Germany

E-mail: justus.lieber@med.uni-tuebingen.de

Abbreviations: HB, hepatoblastoma; SR, standard risk; HR, high risk; MDR, multidrug resistance; CDDP, cisplatin; DOXO, doxorubicin; $\mathrm{BH} 3, \mathrm{Bcl}-2$ homology domain

Key words: hepatoblastoma, multidrug resistance, apoptosis sensitizers, BH3 mimetic drugs, ABT-737, cisplatin, CDDP may allow reduction in CDDP dosages with maintenance of antitumor activity. Sensitizing HB to apoptosis may also render resistant HB susceptible to established chemotherapy regimens.

\section{Introduction}

Hepatoblastoma (HB), the most common primary malignant liver tumor in children, is associated with an excellent outcome [3-year survival $96 \%$ in standard-risk (SR)-HB] due to the effectiveness of combined treatment using chemotherapy and surgery. SR-HB tumors show a high sensitivity to chemotherapy, particularly to the alkylating-like chemotherapeutic agent cisplatin (CDDP) $(1,2)$. Neoadjuvant chemotherapy facilitates tumor resection, and systemic therapy has substantially decreased the incidence of recurrent metastatic disease (3). CDDP as monotherapy is equally effective as combined CDDP/DOXO (doxorubicin) in patients with accurately staged SR-HB regarding complete resection rates. This approach is, as predicted, less toxic than combination treatment regimens $(3,4)$. In contrast, children suffering from high-risk (HR-HB) tumors still present with a poor survival (3-year survival 69\%). A major reason for this fact is multi-drug resistance, which develops in $80 \%$ of initially CDDP- and DOXO-sensitive tumors after 4-5 courses of chemotherapy $(2,5-7)$. Exposure to alternating cycles of CDDP is also associated with clinically significant adverse effects such as irreversible nephrotoxicity, ototoxicity, neurotoxicity and myelosuppression $(8,9)$. Other platin-derived drugs are less toxic but also less effective; therefore, cisplatin remains the agent of choice for HB and many other childhood solid tumors.

Small-molecular sensitizers, which enhance the effects of cytotoxic drugs by sensitizing tumor cells to apoptosis, constitute a promising treatment option for overcoming resistance and improving outcome (10). One of these is ABT-737, an antitumor agent that induces apoptosis by selectively inhibiting the anti-apoptotic proteins $\mathrm{Bcl}-2, \mathrm{Bcl}-\mathrm{XL}$ and $\mathrm{Bcl}-\mathrm{W}$ at the mitochondrial cell level (11). HB cells show overexpression of anti-apoptotic molecules encoded by genes of the Bcl family which play a central role in drug resistance of several types of malignancies including HB $(11,12)$. ABT-737 binds to the $\mathrm{Bcl}-2$ homology domain 3 (BH3) binding groove of Bcl-2 and facilitates the activation of pro-apoptotic Bcl proteins $\mathrm{tBid}$, Bad, Bax and Bim leading to an increase in cytochrome $c$ release and 
an increase in apoptosis. ABT-737 as a single agent has shown activity against several hematopoietic cell lines (leukemia, multiple myeloma and cultured lymphoma) and various solid tumors, including HB in vitro (11,13-18). Highly synergistic in vivo effects have been described when combining ABT-737 with established chemotherapeutic drugs commonly used in treatment protocols of HB, including CDDP $(11,19,20)$.

In this study, we investigated the effects of a combination therapy consisting of ABT-737 and CDDP in a xenograft model of HB.

\section{Materials and methods}

Gene expression analysis. Gene expression analysis was performed using the database E-MEXP-1851 (www.ebi.ac.uk) including 25 samples of $\mathrm{HB}$ and 4 samples of normal liver tissue (21). Differential expression was visualized within the apoptosis pathway using PathVision 2.0.11 (www.pathvision.org).

Drugs. ABT-737 (Abbott GmbH \& Co. KG, Wiesbaden, Germany) was dissolved for in vitro and animal studies as previously described (14). The final concentration in the cell culture was $0.01,0.1,0.3$ and $1 \mu \mathrm{M}$. CDDP was provided by Neocorp AG (Weilheim, Germany).

Tumor cells and culture conditions. The HB cell lines HUH6 (22) and HepT1 (23) were used for all experiments. The cells were transduced with a plasmid encoding Gaussia luciferase (GLuc, pCMV-GLuc; NEB, Frankfurt, Germany). Stable clones were isolated and maintained in DMEM (Gibco BRL, Carlsbad, CA) supplemented with $10 \%$ FCS, G418, $1 \%$ glutamine, and $1 \%$ penicillin/streptomycin (Gibco, Eggenstein, Germany).

Clonogenic assay. Briefly, an equal number of HUH6 and HepT1 cells at a concentration of 10,000 cells/ml were seeded on poly-D-lysine-coated Petri dishes (Becton Dickinson GmbH, Heidelberg, Germany) with $5 \mathrm{ml}$ medium allowing development of cell adhesion for $2 \mathrm{~h}$. Cells were incubated with $0.3 \mu \mathrm{M}$ ABT-737, $1 \mu \mathrm{g} / \mathrm{ml}$ CDDP or a combination of both drugs for $96 \mathrm{~h}$. The medium was then replaced, and colonies were washed, fixed with glutaraldehyde $(6.0 \% \mathrm{v} / \mathrm{v})$, and stained with crystal violet $(0.5 \% \mathrm{w} / \mathrm{v})$. The cell colonies growing on a surface of $125 \mathrm{~mm}^{2}$ in different regions of the culture plate were counted using a stereomicroscope (Axioscope 40; Carl Zeiss, Oberkochen, Germany). A colony was defined to consist of at least 50 cells. All studies were performed in triplicates.

Animals and xenotransplantation. Xenotransplantation was performed as previously described (24). All animal studies were conducted according to criteria outlined in the 'Guide for the Care and Use of Laboratory Animals' [Animal Care and Use: Policy Issues in the 1990's, National Institutes of Health/Office for the Protection from Research Risks (NIH/ OPRR), 1989. Proceedings of NIH/OPRR Conference, Bethesda, MD], and were approved by the local government's ethics authority for animal experiments (Regierungspräsidium Tübingen, Referat 35, number CK1/09). HUH6 cells were injected into the flank of 6- to 8-week-old NOD/LtSz-scid IL2R $\gamma$ null mice (NSG). For each tumor $0.2 \mathrm{ml}$ of tumor cell suspension $\left(2 \times 10^{6}\right.$ cells) was injected subcutaneously into the paravertebral areas. The observation time was 4 weeks. Each group consisted of 5-6 animals. Treatment was initiated when tumors had reached a length of $5 \mathrm{~mm}$. CDDP in a $200 \mu \mathrm{l}$ saline solution was administered i.p. once per day on days 1-4 with a dosage of $1.25 \mathrm{mg} / \mathrm{kg}$ (low dose) or $3.0 \mathrm{mg} / \mathrm{kg}$ body weight (high dose), respectively. ABT-737 was injected i.p. with a dosage of $100 \mathrm{mg} / \mathrm{kg}$ body weight alone or in combination with CDDP using the same schedule. Control animals were left untreated until day 10 unless the tumor volume exceeded $1 \mathrm{~cm}^{3}$. Tumor volumes $(\mathrm{V}=4 / 3 \pi \times \mathrm{a} / 2 \mathrm{x} \mathrm{b} / 2 \mathrm{x} \mathrm{c} / 2)$ and body weight of all animals were determined daily. Relative tumor growth was calculated as the percentage of tumor volume at each time point compared to day 0 . Blood samples were obtained from the retrobulbar plexus on days 0 and 10. Serum GLuc activity was quantified in fresh serum. Therefore, $5 \mu \mathrm{l}$ of serum was added to $50 \mu \mathrm{l}$ Gaussia GlowJuice (J.P.K. Instruments AG, Berlin, Germany), and GLuc activity was measured using a luminometer (Magic ${ }^{\circledR}$ Lite Analysator, Ciba Corning) after adding $1 \mu \mathrm{l}$ coelenterazine $(100 \mu \mathrm{M})$ to acquire photon counts for $10 \mathrm{sec}$. Activity was expressed as relative light units per second (RLU/sec). Tumors were explanted on day 10 and prepared for histological analysis.

Histology and immunohistochemistry. Paraffin-embedded sections obtained from xenografted tumors were used for immunohistochemical staining against Ki-67 (mouse antihuman Ki-67, 1:400; Dako GmbH, Hamburg, Germany) as previously described (14). The proliferation index was expressed as the mean \pm SD of positively stained cells per microscopic field of two regions in each tumor of the treatment groups.

Statistical analysis. Statistical analysis of clonogenicity of HB cells was carried out using two-way ANOVA followed by Bonferroni post-test using GraphPad Prism 4.00 (GraphPad Software, San Diego, CA, USA, www.graphpad.com). Relative tumor growth and body weights for each group of animals were compared to controls using the Student's t-test. Data plotted on graphs represent the means \pm SD. Significance was assumed for all p-values $<0.05$.

\section{Results}

Expression of pro- and anti-apoptotic proteins in $\mathrm{HB}$ and normal liver tissue. Proteins involved in apoptosis modulation and signalling were visualized using differential gene expression (Fig. 1). Death receptors of the TNF-family generally were downregulated in tumors or showed equal expression levels when compared to normal liver tissue. Caspases, such as CASP8 and CASP3, were highly expressed in tumors. Expression levels of the anti-apoptotic Mcl-1 were high in normal liver tissue and only slightly reduced in tumors; however, still high expression levels were found. Other antiapoptotic proteins, such as Bcl-XL, Bcl-W and survivin were highly expressed in tumors, whereas pro-apoptotic proteins, such as BAD and PUMA showed low expression levels. However, Bax, as a promoter of apoptosis, was present. BAK-1 was equally expressed in tumors and normal liver tissue. Taken together, an anti-apoptotic state predominated in the HB tumors compared to normal liver tissue. Consequently, the 


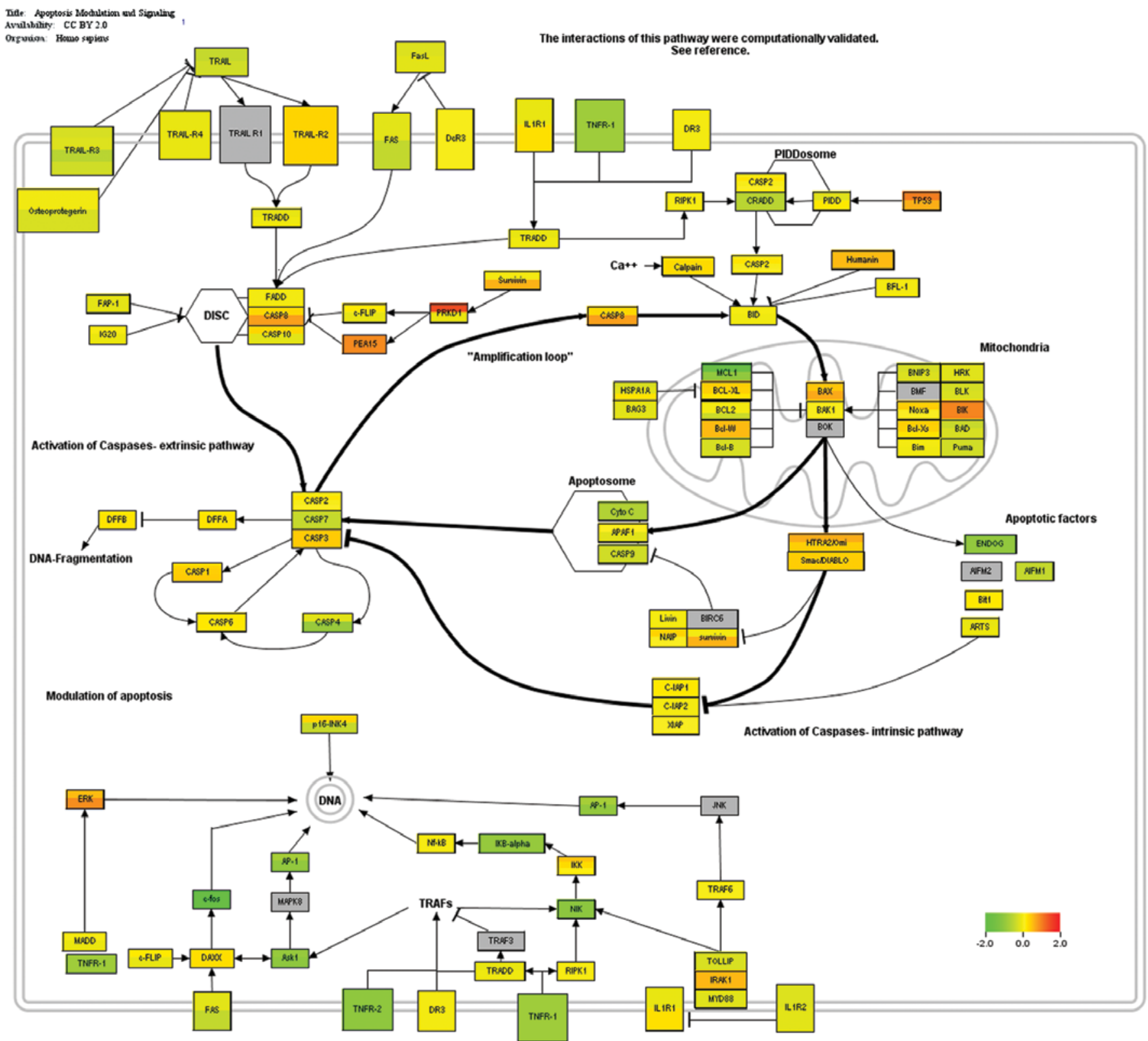

Figure 1. Differential expression of genes involved in the modulation and signaling of apoptosis. Red colors represent high expression of genes in tumors related to normal liver tissue, and green colors represent low expression of genes in tumors related to normal liver tissue.

use of apoptosis modulators or sensitizers constitutes a promising option in order to enhance the effects of drugs acting on induction of the apoptosis cascade.

Additive effects of CDDP and ABT-737 in HB cells. ABT-737 enhances the effect of various cytotoxic drugs in the combination treatment of tumor cell lines. To determine the effects of the treatment on HB cells, a clonogenity assay was carried out. CDDP alone led to a decreased number of clones in HepT1 and HUH6 cells (Fig. 2). The reduction in clone number following ABT-737 treatment was inferior to the effect of CDDP and was not significantly different from the control experiments. CDDP in combination with ABT-737 significantly decreased the number of clones in HB cell cultures $(p<0.05)$. The number of clones was more than 5 -fold reduced following the combination treatment compared to treatment with CDDP alone. In general, HUH6 cells showed higher sensitivity to CDDP as well as the combined treatment.

Treatment of $H B$ xenografts with a combination of $C D D P$ and $A B T-737$. HUH6 xenografts were used to describe the effects of ABT-737 in combination with CDDP in vivo. All of the xenotransplanted animals developed measurable tumors after 4 weeks. Relative tumor volumes showed maximal growth in the control group $(n=5)$ (Fig. 3A). Tumor volumes increased three times within 4 days. In the group treated with low dose CDDP $(1.25 \mathrm{mg} / \mathrm{kg}$ for 4 consecutive days; $\mathrm{n}=6)$ the relative tumor volume was duplicated. Significantly reduced tumor growth was observed in mice after combined treatment $(n=5)$ compared to treatment with CDDP alone $(\mathrm{p}<0.02)$. In the group administered the combined treatment, two mice did not show tumor growth at all and two tumors shrank during treatment. 
A

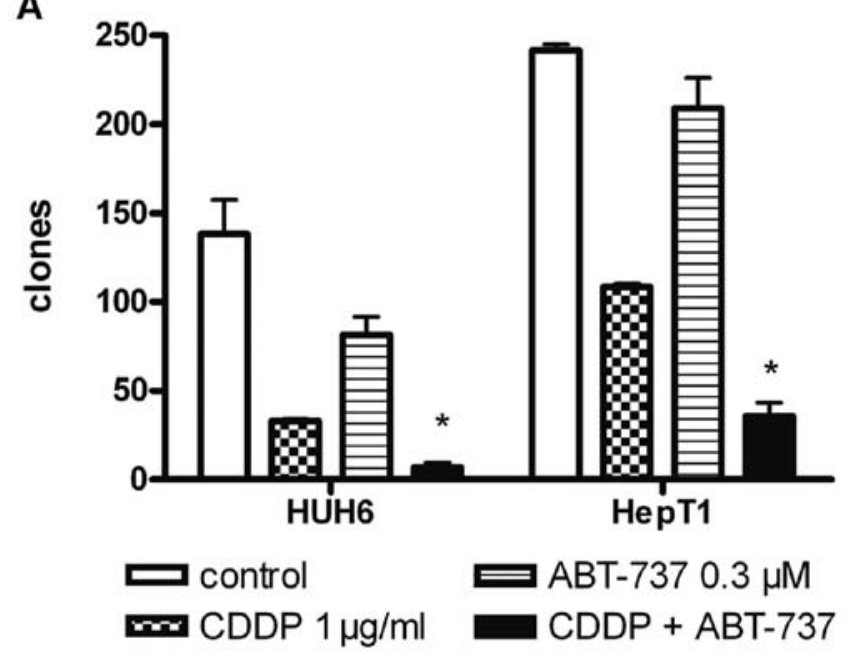

B

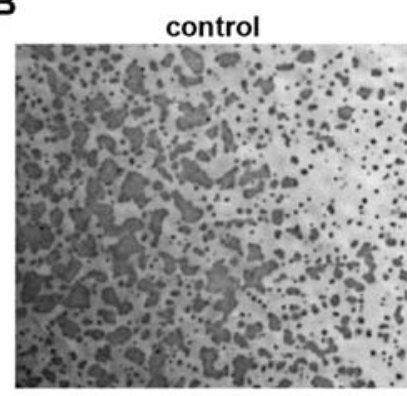

ABT-737

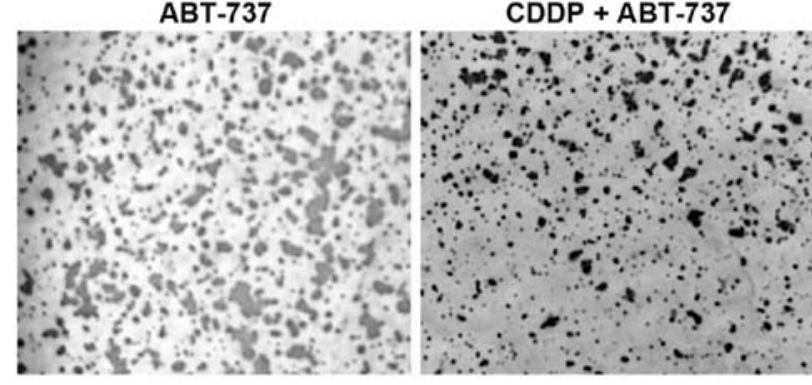

Figure 2. Clonogenicity of HB cells. HB cells were treated with ABT-737 and CDDP. HUH6 and HepT1 cells were incubated in triplicates with $0.3 \mu \mathrm{M}$ ABT-737 and $1 \mu \mathrm{g} / \mathrm{ml} \mathrm{CDDP}$ for $24 \mathrm{~h}$. The number of clones was determined $96 \mathrm{~h}$ later in a clonogenicity assay (means $\pm \mathrm{SD}$ ). Significantly lower number of clones was detected in cells following the combined treatment compared with the controls and monotherapy treatment $\left({ }^{*} \mathrm{p}<0.05\right.$, two-way ANOVA, Bonferroni post-test) (A). Representative images of HUH6 cultures (B).

As an additional control parameter, expression of the transgene GLuc was detected in the blood of all mice at levels > $200 \mathrm{RLU} /$ sec, revealing tumor growth (Fig. 3B). Relative GLuc activity increased during the experiment in the controls and in mice under treatment.

Toxicity was monitored by changes in body weight during treatment. Tumor-bearing mice in the control group gained weight or remained constant during the experiment (Fig. 4). Loss of $>10 \%$ body weight was observed in the group treated with high dose CDDP ( $3 \mathrm{mg} / \mathrm{kg}$ body weight) compared to control animals. However, body weights remained stable after treatment with low dose CDDP $(1.25 \mathrm{mg} / \mathrm{kg})$. Treatment with a combination of ABT-737 and high dose CDDP led to death in 4 of 6 animals, whereas 3 of 5 animals survived the experiment
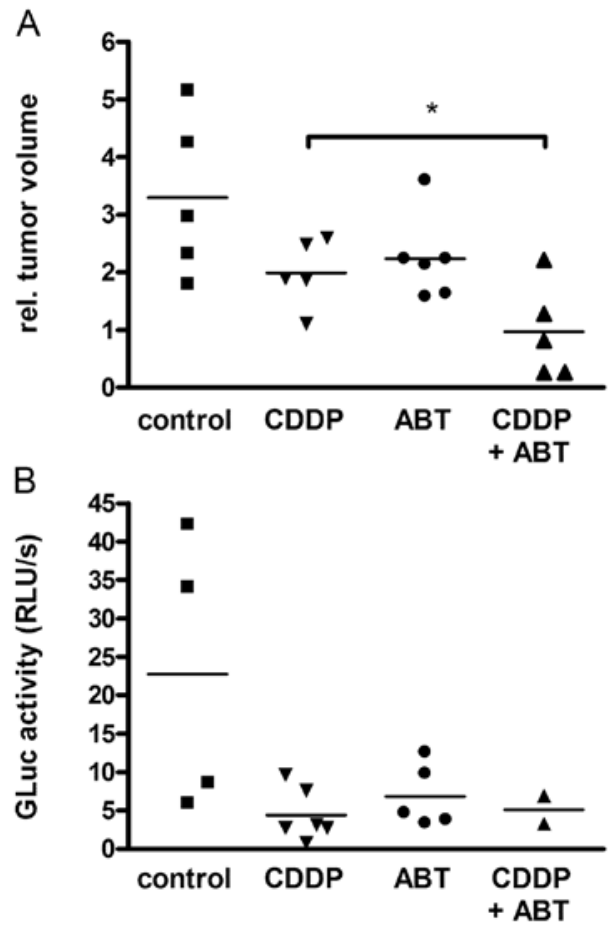

Figure 3. Development of HUH6 tumors and GLuc activity in mice. Mice with xenotransplanted HUH6 tumors were treated with ABT-737 $(100 \mathrm{mg} / \mathrm{kg}$ body weight) and CDDP (1.25 mg/kg) for 4 consecutive days. Relative tumor volume for each animal indicated tumor growth except in four mice treated with the combination of the drugs. Significantly reduced tumor growth was observed in mice after combined treatment compared to monotherapy at day 9 ( ${ }^{*} \mathrm{p}<0.02$, Student's t-test) (A). Relative GLuc activity was increased in all animals indicating tumor growth (B).

when treated with combined low dose CDDP. No toxcitiy was observed after treatment with ABT-737 alone.

Histological analysis of HB xenografts of the control group and ABT-737 group showed high density of tumor cells in H\&E staining (Fig. 5). Fewer vital tumor cells and small areas of necrosis were noted in xenografts of the CDDP group. After combined treatment, multiple picnotic cells, hemorrhagic infarction, and large areas of necrosis were noted in the tumors. In this group, large areas of disintegrated tumors were detected. Proliferation rates were evaluated by anti-Ki-67 staining and by mean proliferation index \pm SD. Comparable proliferation was detected in the control tumors $(699 \pm 108)$, tumors treated with low dose CDDP $(745 \pm 170)$, and ABT-737 alone (715 \pm 98$)$. Cell proliferation was significantly reduced after combined treatment using ABT-737 and low dose CDDP $(475 \pm 231)$ when compared to all other groups $(\mathrm{p}<0.5$, ANOVA with Bonferroni's multiple comparison test).

Taken together, additive effects following CDDP and ABT-737 treatment were demonstrated in HB xenografts by assessing tumor growth and histological appearance.

\section{Discussion}

Complete tumor resection is mandatory for the survival of patients suffering from HB. However, $50 \%$ of tumors are primarily unresectable or metastases are presented at diagnosis $(6,25)$. Therefore, the European Study Groups for Liver Tumors in Children recommend surgery after neoadjuvant 

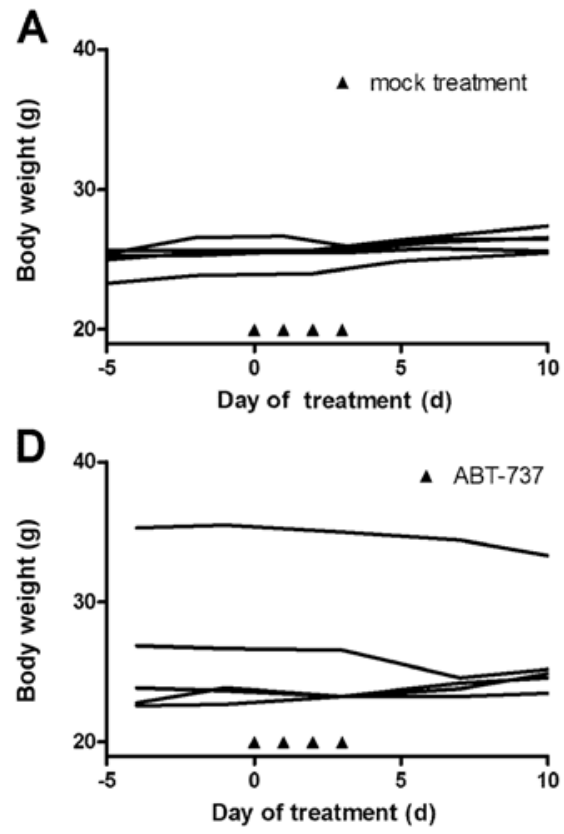
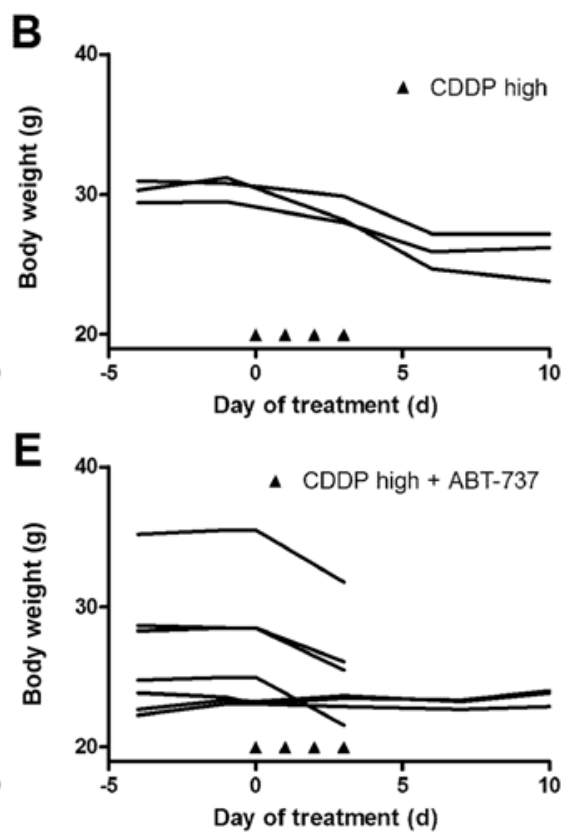
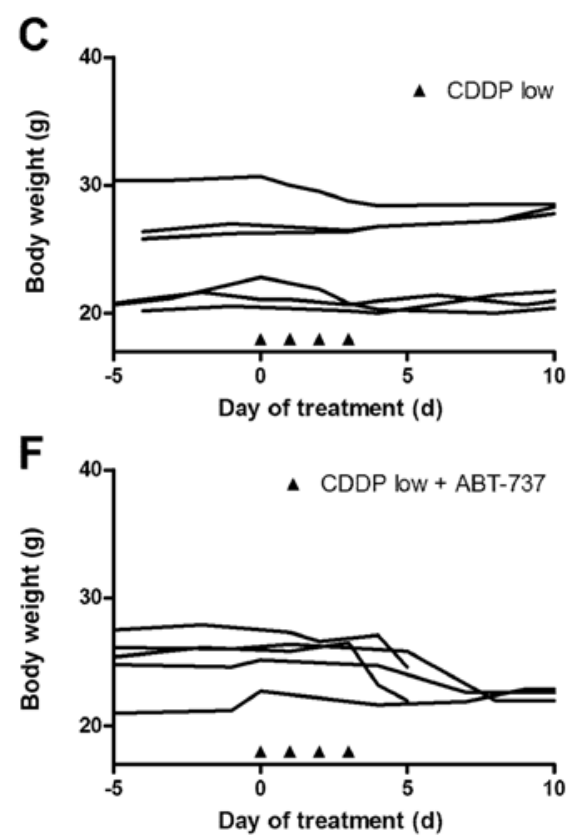

Figure 4. Development of body weight in NSG mice. NSG mice were treated as indicated on 4 consecutive days. The body weight is shown for each individual mouse at indicated time points of the experiment. Loss of body weight was observed in the group treated with CDDP 'high dose' (3 mg/kg) (B) compared with control animals (A). Body weights remained stable in the group treated with CDDP 'low dose' (1.25 mg/kg) (C). Four of 6 animals treated with a combination containing 'high' CDDP died after the treatment cycle (E), whereas 3 of 5 animals survived the experiment when treated with a combination containing 'low' CDDP (F). Treatment with ABT-737 alone had no significant effect on body weight of the mice (D).

chemotherapy. HB exhibits a good response to neoadjuvant chemotherapy resulting in reduced tumor size and better resectability. CDDP is the most important cytotoxic agent, particularly in SR-HB, and CDDP treatment leads to an excellent 3-year survival rate of $96 \%$ (2). Various regimens include the use of carboplatin in addition to CDDP in order to improve treatment efficacy. However, results were not improved, and therapy was associated with significantly more toxicity, even though amifostine had been used as a cytoprotective adjuvant (5). CDDP/DOXO (PLADO) treatment was also not superior compared to CDDP monotherapy in SR-HB (2). For HR-HB, intensification of neoadjuvant chemotherapy with carboplatin/DOXO alternating with CDDP was recommended in the SIOPEL 3 study, and this treatment showed tolerable toxicity together with effective tumor reduction. Shortening the time intervals between the courses is planned in the SIOPEL 4 for the group of high-risk patients to further intensify chemotherapy (7). This may increase the risk of toxicity. Collectively, treatment results of extended and metastatic HR-HB are still not satisfactory irrespective of the principle treatment strategy and applied drug combination. This was also observed and described by the first Japanese Hepatoblastoma Trial (JPLT-1) and the current German GPOH-HB99 study $(6,7,26)$. In addition, multidrug resistance complicates the response to chemotherapy and develops in $80 \%$ of patients initially sensitive to CDDP and DOXO after 4-5 courses of chemotherapy (27). In order to improve the efficacy of chemotherapeutic agents several mechanisms are currently under investigation. Modulation of apoptosis using small BH3 mimetic molecules, such as ABT-737, is one such mechanism $(10,15)$.

CDDP acts as an alkylating agent and finally induces apoptosis following two pathways. It activates reactive oxygen species (ROS), which represent a specific vulnerability of malignant cells (28). However, this pathway is inhibited by anti-apoptotic $\mathrm{Bcl}-2$ protein, an important member of the Bcl family. Various malignancies, such as $\mathrm{HB}$, demonstrate $\mathrm{Bcl}-2$ overexpression, which plays a central role in resistance to chemotherapy (29). In contrast, CDDP increases p53 levels, which activate proapoptotic NOXA, PUMA, Bax and p38. CDDP activates the apoptotic pathway and MAP kinase signaling (30). In rapidly proliferating tumor cells, chemotherapeutics may be more effective at physiological apoptosis homeostasis than in this observed anti-apoptotic state. Therefore, we used lower dosages of CDDP in combination with the $\mathrm{BH} 3$ mimetic ABT-737 to facilitate induction of apoptosis through ROS.

ABT-737 induces apoptosis as a single drug when treating various cell lines and has also previously shown additive effects in HB cell lines when combined with various cytotoxic drugs despite the expression of Mcl-1 $(11,16,19,20,31)$. In a previous study, we obtained similar results with a combination therapy consisting of ABT-737 and paclitaxel in HB xenografts (14). In the present study, CDDP also led to a tumor response in xenografts; however, we observed only reduced growth rates, but not shrinkage of xenografts compared to initial tumor volume. Additive effects were observed after a combined therapy using ABT-737 and CDDP, resulting in inhibition of tumor growth and in certain cases a reduction in tumor volume. Histologically, we found noticeably less vital tumor cells in HB xenografts after combination therapy and large areas of disintegrated tumors were detected.

In this study, high doses of CDDP (3 mg/ $\mathrm{kg}$ ) led to significant toxicity in NOD mice, whereas low doses of CDDP $(1.25 \mathrm{mg} / \mathrm{kg})$ showed transient toxic effects. Clinically significant adverse effects associated with CDDP exposure in human patients are well described including irreversible nephrotoxicity, ototoxicity, neurotoxicity and myelosuppression (8). 

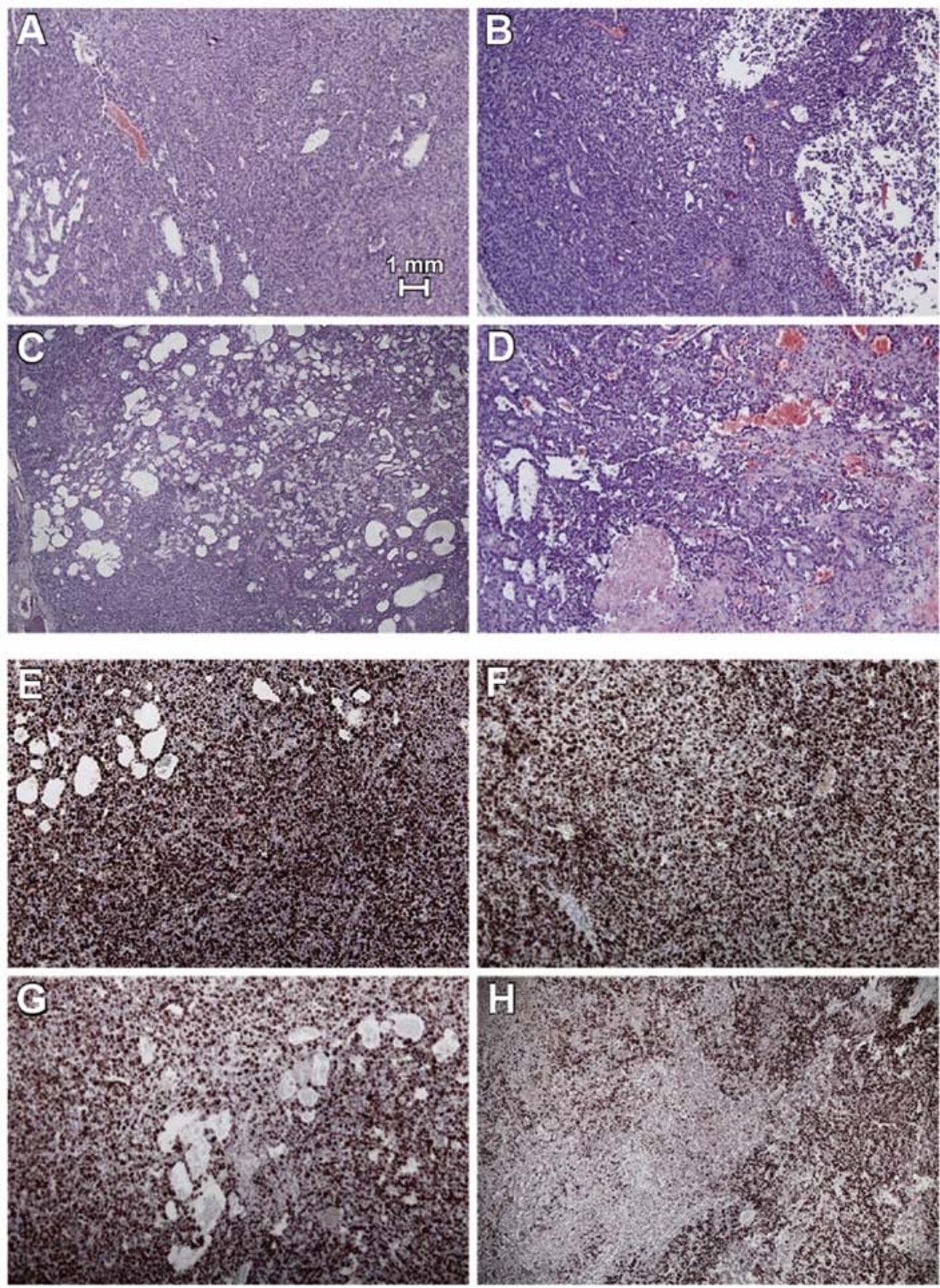

Figure 5. Immunhistological analysis of HB xenografts. Images show staining of hematoxylin and eosin (A-D) and detection of Ki-67 (E-H). Representative samples are shown for controls (A and E), ABT-737 (B and F), CDDP (C and G), and combined treatment (D and H). Combined treatment using CDDP and ABT-737 revealed high tissue damage with multiple picnotic nuclei and necrotic tissue destruction.

Thus, lowering the dosage of CDDP along with treatment in combination with ABT-737, which demonstrates significantly reduced tumor growth compared to CDDP monotherapy in vivo, may also maintain or even enhance antitumor activity in patients. In contrast to CDDP, ABT-737 alone $(100 \mathrm{mg} /$ $\mathrm{kg}$ body weight) was not associated with toxic effects in this and other studies $(15,19,20)$. In this context, we assume the toxicity to be mouse strain independent, as has been previously described in nude mice NMRI (nu/nu) treated with CDDP alone (32). When combining CDDP and ABT-737, the toxicity was even higher than that following treatment with CDDP alone. To reduce toxicity due to combination treatment, second generation orally bioavailable $\mathrm{BH} 3$ mimetics, such as ABT-263, may be used (33). In addition, other BH3 mimetic drugs have been considered to be attractive candidates for combination treatments. Currently, obatoclax is under investigation in several clinical trials including those targeting solid tumor malignancies and has been described to be well tolerated without dose-limiting toxicity (34-36). Obatoclax has already shown additive effects in the treatment of HB cells when combined with CDDP and therefore will be assessed in further optimization studies (15). Finally, the most important advantage of combination treatment is dose reduction of both the cytostatic and the $\mathrm{BH} 3$ mimetic drug, reducing side effects while maintaining antitumor activity. Based on this assumption, problematic thrombocytopenia after combination treatment may be weakened, as it is only described with high dosages of $\mathrm{BH} 3$ mimetic drugs $(33,37,38)$.

However, apoptosis sensitizers may be active only in those patients with a high anti-apoptotic state, as particularly high 
deviations of means were observed in the gene expression analyses. These findings may recommend the evaluation of the apoptosis status of patients during the initial treatment phase, e.g. using an array for apoptosis (11). Based on our results, a combination therapy of CDDP and $\mathrm{BH} 3$ mimetic drugs may serve as a promising addition to the treatment of advanced $\mathrm{HB}$ in clinical settings.

In conclusion, the primary goal of current chemotherapy in HB is reduction of tumor volume and vitality in order to enable complete surgical resection. Our results have confirmed the optimization of chemotherapy by using modulators of apoptosis. CDDP, which is the most commonly used cytotoxic agent in most trials of $\mathrm{HB}$, reduces tumor growth when combined with ABT-737. Sensitizing HB cells to apoptosis may also restore the sensitivity of resistant HB to established therapeutic regimens.

\section{Acknowledgements}

The authors wish to acknowledge Abbott Laboratories for providing ABT-737.

\section{References}

1. Loehrer PJ and Einhorn LH: Drugs five years later. Cisplatin. Ann Intern Med 100: 704-713, 1984.

2. Perilongo G, Maibach R, Shafford E, et al: Cisplatin versus cisplatin plus doxorubicin for standard-risk hepatoblastoma. N Engl J Med 361: 1662-1670, 2009.

3. Pritchard J, Brown J, Shafford E, et al: Cisplatin, doxorubicin, and delayed surgery for childhood hepatoblastoma: a successful approach - results of the first prospective study of the International Society of Pediatric Oncology. J Clin Oncol 18: 3819-3828, 2000

4. Sullivan MJ: Hepatoblastoma, cisplatin, and ototoxicity: good news on deaf ears. Cancer 115: 5623-5626, 2009.

5. Ortega JA, Douglass EC, Feusner JH, et al: Randomized comparison of cisplatin/vincristine/fluorouracil and cisplatin/ continuous infusion doxorubicin for treatment of pediatric hepatoblastoma: A report from the Children's Cancer Group and the Pediatric Oncology Group. J Clin Oncol 18: 2665-2675, 2000.

6. Perilongo G, Shafford E, Maibach R, et al: Risk-adapted treatment for childhood hepatoblastoma. Final report of the second study of the International Society of Paediatric Oncology - SIOPEL 2. Eur J Cancer 40: 411-421, 2004.

7. Zsiros J, Maibach R, Shafford E, et al: Successful treatment of childhood high-risk hepatoblastoma with dose-intensive multiagent chemotherapy and surgery: final results of the SIOPEL-3HR study. J Clin Oncol 28: 2584-2590, 2009.

8. Cvitkovic E: Cumulative toxicities from cisplatin therapy and current cytoprotective measures. Cancer Treat Rev 24: 265-281, 1998.

9. Knight KR, Kraemer DF and Neuwelt EA: Ototoxicity in children receiving platinum chemotherapy: underestimating a commonly occurring toxicity that may influence academic and social development. J Clin Oncol 23: 8588-8596, 2005.

10. Chonghaile TN and Letai A: Mimicking the BH3 domain to kill cancer cells. Oncogene 27 (Suppl 1): 149-157, 2008.

11. Lieber J, Kirchner B, Eicher C, et al: Inhibition of Bcl-2 and Bcl-X enhances chemotherapy sensitivity in hepatoblastoma cells. Pediatr Blood Cancer 55: 1089-1095, 2010.

12. Adesina AM, Lopez-Terrada D, Wong KK, et al: Gene expression profiling reveals signatures characterizing histologic subtypes of hepatoblastoma and global deregulation in cell growth and survival pathways. Hum Pathol 40: 843-853, 2009.

13. Konopleva M, Contractor R, Tsao T, et al: Mechanisms of apoptosis sensitivity and resistance to the BH3 mimetic ABT-737 in acute myeloid leukemia. Cancer Cell 10: 375-388, 2006.

14. Lieber J, Eicher C, Wenz J, et al: The BH3 mimetic ABT-737 increases treatment efficiency of paclitaxel against hepatoblastoma. BMC Cancer 11: 362, 2011.

15. Lieber J, Ellerkamp V, Wenz J, et al: Apoptosis sensitizers enhance cytotoxicity in hepatoblastoma cells. Pediatr Surg Int 28: 149-159, 2012.
16. Oltersdorf T, Elmore SW, Shoemaker AR, et al: An inhibitor of Bcl-2 family proteins induces regression of solid tumours. Nature 435: 677-681, 2005.

17. Trudel S, Stewart AK, Li Z, et al: The Bcl-2 family protein inhibitor, ABT-737, has substantial antimyeloma activity and shows synergistic effect with dexamethasone and melphalan. Clin Cancer Res 13: 621-629, 2007.

18. van Delft MF, Wei AH, Mason KD, et al: The BH3 mimetic ABT-737 targets selective Bcl-2 proteins and efficiently induces apoptosis via Bak/Bax if Mcl-1 is neutralized. Cancer Cell 10: 389-399, 2006.

19. High LM, Szymanska B, Wilczynska-Kalak U, et al: The Bcl-2 homology domain 3 mimetic ABT-737 targets the apoptotic machinery in acute lymphoblastic leukemia resulting in synergistic in vitro and in vivo interactions with established drugs. Mol Pharmacol 77: 483-494, 2010.

20. Mason KD, Khaw SL, Rayeroux KC, et al: The BH3 mimetic compound, ABT-737, synergizes with a range of cytotoxic chemotherapy agents in chronic lymphocytic leukemia. Leukemia 23: 2034-2041, 2009.

21. Cairo S, Armengol C, De Reynies A, et al: Hepatic stem-like phenotype and interplay of Wnt/beta-catenin and Myc signaling in aggressive childhood liver cancer. Cancer Cell 14: 471-484, 2008.

22. Doi I: Establishment of a cell line and its clonal sublines from a patient with hepatoblastoma. Gann 67: 1-10, 1976.

23. Pietsch T, Fonatsch C, Albrecht S, Maschek H, Wolf HK and von Schweinitz D: Characterization of the continuous cell line HepT1 derived from a human hepatoblastoma. Lab Invest 74: 809-818, 1996.

24. Warmann SW, Heitmann H, Teichmann B, et al: Effects of P-glycoprotein modulation on the chemotherapy of xenotransplanted human hepatoblastoma. Pediatr Hematol Oncol 22: 373-386, 2005.

25. Reynolds M: Conversion of unresectable to resectable hepatoblastoma and long-term follow-up study. World J Surg 19: 814-816, 1995.

26. Haberle B, Bode U and von Schweinitz D: Differentiated treatment protocols for high- and standard-risk hepatoblastoma - an interim report of the German Liver Tumor Study HB99. Klin Padiatr 215: 159-165, 2003 (In German).

27. von Schweinitz D, Byrd DJ, Hecker H, et al: Efficiency and toxicity of ifosfamide, cisplatin and doxorubicin in the treatment of childhood hepatoblastoma. Study Committee of the Cooperative Paediatric Liver Tumour Study HB89 of the German Society for Paediatric Oncology and Haematology. Eur J Cancer 33: 1243-1249, 1997.

28. Roux PP and Blenis J: ERK and p38 MAPK-activated protein kinases: a family of protein kinases with diverse biological functions. Microbiol Mol Biol Rev 68: 320-344, 2004.

29. Warmann SW, Frank H, Heitmann H, et al: Bcl-2 gene silencing in pediatric epithelial liver tumors. J Surg Res 144: 43-48, 2008.

30. Ono $\mathrm{K}$ and Han J: The p38 signal transduction pathway: activation and function. Cell Signal 12: 1-13, 2000.

31. Chauhan D, Velankar M, Brahmandam M, et al: A novel Bcl-2/ $\mathrm{Bcl}-\mathrm{X}(\mathrm{L}) / \mathrm{Bcl}-\mathrm{w}$ inhibitor ABT-737 as therapy in multiple myeloma. Oncogene 26: 2374-2380, 2007.

32. Warmann S, Hunger M, Teichmann B, Flemming P, Gratz KF and Fuchs J: The role of the MDR1 gene in the development of multidrug resistance in human hepatoblastoma: clinical course and in vivo model. Cancer 95: 1795-1801, 2002.

33. Tse C, Shoemaker AR, Adickes J, et al: ABT-263: a potent and orally bioavailable Bcl-2 family inhibitor. Cancer Res 68: 3421-3428, 2008.

34. Hwang JJ, Kuruvilla J, Mendelson D, et al: Phase I dose finding studies of obatoclax (GX15-070), a small molecule pan-BCL-2 family antagonist, in patients with advanced solid tumors or lymphoma. Clin Cancer Res 16: 4038-4045, 2010.

35. Paik PK, Rudin CM, Brown A, et al: A phase I study of obatoclax mesylate, a Bcl-2 antagonist, plus topotecan in solid tumor malignancies. Cancer Chemother Pharmacol 66: 1079-1085, 2010.

36. Schimmer AD, O'Brien S, Kantarjian $\mathrm{H}$, et al: A phase I study of the pan bcl-2 family inhibitor obatoclax mesylate in patients with advanced hematologic malignancies. Clin Cancer Res 14: 8295-8301, 2008

37. Mason KD, Carpinelli MR, Fletcher JI, et al: Programmed anuclear cell death delimits platelet life span. Cell 128: 1173-1186, 2007.

38. Zhang H, Nimmer PM, Tahir SK, et al: Bcl-2 family proteins are essential for platelet survival. Cell Death Differ 14: 943-951, 2007. 